\title{
Utilization of public health care by people with private health insurance: a systematic review and meta-analysis
}

\author{
Congcong Zhang ${ }^{1}$, Chenwei $\mathrm{Fu}^{1}$, Yimin Song ${ }^{1}$, Rong Feng ${ }^{1}$, Xinjuan $\mathrm{Wu}^{2^{*}}$ and Yongning $\mathrm{Li}^{\mathrm{i}^{*}}$
}

\begin{abstract}
Background: The objective of this systematic review was to explore the association between private health insurance and health care utilization.

Methods: We searched the MEDLINE, EMBASE, and Cochrane Central Register of Controlled Trials (CENTRAL) electronic databases for relevant articles since 2010. Studies were eligible if they described original empirical research on the utilization of public health care by individuals with private health insurance, compared with individuals without private insurance. A pooled measure of association between insurance status with health care utilization was assessed through meta-analysis.

Results: Twenty-six articles were included in the final analysis. We found that patients with private insurance did not use more public health care than people without private insurance $(P<0.05)$. According to the subgroup analysis, people with private insurance were more likely to be hospitalized than people with no insurance (OR 1.67; $95 \% \mathrm{Cl}, 1.18$ to 2.36$)$.

Conclusions: People with private insurance did not increase their use of health care (outpatient services), compared to those without private insurance. Private health insurance coverage may ease the financial burden on patients and on the public health insurance system.
\end{abstract}

Keywords: Private health insurance, Health care utilization, Systematic review, Meta-analysis

\section{Background}

As most countries across the world face rapidly escalating health expenditures, exorbitant out-of-pocket payments have resulted in high demand for supplementary private health insurance [1, 2]. For instance, in 2015, approximately $80 \%$ of households in the United States had to purchase at least one private health insurance plan, and more than $25 \%$ of Brazilians had private health insurance in 2019 [3, 4].

\footnotetext{
*Correspondence: Wuxinjuan@pumch.cn; pumchcongcong@126.com ${ }^{2}$ Department of Nursing, Peking Union Medical College Hospital, No.1 Shuaifuyuan, Wangfujing Dongcheng District, Beijing 100730, China ${ }^{1}$ Department of International Medical Servicers, Peking Union Medical College Hospital, No.1 Shuaifuyuan, Wangfujing Dongcheng District, Beijing 100730, China
}

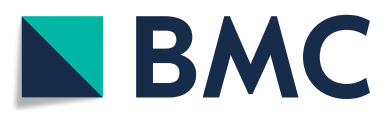

(c) The Author(s). 2020 Open Access This article is licensed under a Creative Commons Attribution 4.0 International License, which permits use, sharing, adaptation, distribution and reproduction in any medium or format, as long as you give appropriate credit to the original author(s) and the source, provide a link to the Creative Commons licence, and indicate if changes were made. The images or other third party material in this article are included in the article's Creative Commons licence, unless indicated otherwise in a credit line to the material. If material is not included in the article's Creative Commons licence and your intended use is not permitted by statutory regulation or exceeds the permitted use, you will need to obtain permission directly from the copyright holder. To view a copy of this licence, visit http://creativecommons.org/licenses/by/4.0/ The Creative Commons Public Domain Dedication waiver (http://creativecommons.org/publicdomain/zero/1.0/) applies to the data made available in this article, unless otherwise stated in a credit line to the data.

The role of private health insurance is fiercely debated. Some researchers believe that the use of private health insurance should be encouraged in order to ease the financial burden on patients and on social healthcare systems [5]. However, others maintain that the use of private health insurance will contribute to the current rapid increase in health expenditures, induce fragmentation of the healthcare system, and aggravate social inequity by increasing the gap in health care utilization between opposite ends of the socioeconomic spectrum [6,7]. One of the critical controversies over private health insurance is its potential impact on health care utilization. If individuals with private health insurance increase their utilization of health care, the result will be inequity in health care 
utilization between those who purchase private health insurance and those who do not.

Although previous studies have examined the effect of private insurance on the utilization of public health care in specific countries $[3,6]$, no study published to date has systematically investigated the issue on a global scale. However, it is necessary for stakeholders to understand the role that private insurance companies play the use of healthcare services from a macro perspective. The objective of this systematic review was to synthesize available evidence to compare the effect of private health insurance to the effect of having no (private) insurance or public health care insurance on the utilization of health care (inpatient and outpatient resources) among all kinds of patients worldwide.

\section{Methods}

\section{Search strategy}

This systematic review was performed in accordance with the recommendations of the Preferred Reporting Items for Systematic Reviews and Meta-Analyses (PRISMA) guidelines, but the review protocol was not registered [8]. Two reviewers searched MEDLINE, EMBASE, and the Cochrane Central Register of Controlled Trials (CENTRAL) databases for relevant articles published from January 1, 2010 to June 1, 2019. The search terms used included: "health insurance," "private or commercial health plan(s)," "private or commercial health insurance," "private or commercial health company," "health within six words around the word of utilization or utility," and "hospital within six words around the word of utilization or utility" (see detailed search strategies in Additional file 1). We searched for additional references by cross-checking the reference lists of the studies retrieved and of relevant reviews. We also contacted researchers in the field to identify trials that were eligible for inclusion.

\section{Inclusion and exclusion criteria}

We included both prospective and retrospective longitudinal controlled studies in this systematic review. Studies were eligible if they described original empirical research on the utilization of health care by individuals with private health insurance. The eligibility criteria were: 1 ) original studies (randomized controlled trial, case-control, cohort, cross-sectional, or pre-post); 2) one group of study participants with private health insurance (exposure group); 3) one group of study participants without private insurance (control); 4) utilization of health care [outpatient services: emergency department (ED) visits, clinic visits; inpatient services: length of stay (LOS), hospitalization rate] as an outcome [9]; 5) publication in the English language in 2010 or later. Reviews, commentaries, protocols, editorials, case reports, qualitative research, and letters were excluded. Studies on diagnostic support (e.g., radiology, clinical pathology) were also excluded. If two articles were found to derive from the same study, only the original study was included. However, if different target outcomes were reported, then both papers were included.

\section{Study selection}

Titles and abstracts were first screened for relevance by two independent reviewers, and full-text articles with potential eligibility were downloaded for further assessment. When consensus could not be reached, disagreements were resolved by consulting a third author.

\section{Data collection}

Data were collected with an extraction form validated in pilot studies. The data items extracted in this review were as follows: (1) The surname of the first author with the year in which the paper was published; (2) study design; (3) country in which the study was conducted; (4) full report or abstract; (5) target population; (6) target exposure group; (7) target control group; (8) target outcomes [emergency department (ED) visits, clinic visits, length of stay (LOS), and/or hospitalization rate]; (9) the numerical data included the number of visits to the ED, the percentages of visits to the ED, the rates of hospitalization, the rates of outpatient office visits, and the length of inpatient stays (days).

\section{Quality assessment}

Risk of bias was assessed independently by two reviewers. We applied the ROBINS I tool to assess the risk of bias among non-randomized intervention studies [10]. Risk of bias was assessed at the study level, and these results were used to inform a GRADE evidence assessment [11].

\section{Statistical analysis}

We performed meta-analyses of the studies to obtain a pooled estimate for the utilization of health care by individuals with private health insurance, compared with individuals without private health insurance. Odds ratios [with 95\% confidence intervals (CIs)] were obtained for the rates of visits to the ED, the percentages of ED visits, and the rates of hospitalization with Review Manager 5.3 software [12]. Using the same software program, mean differences were obtained for the rates of outpatient office visits and the length of inpatient stay (days). Pvalues $<0.05$ were considered as statistically significant. Between-study heterogeneity was measured using Cochrane's Q-test and the Higgins $I^{2}$ statistic $(P<0.10$ or $I^{2}>50 \%$ ) was considered as statistically significant heterogeneity [13]. When heterogeneity was present, a random-effect model (Der Simonian and Laird method) was applied. The fixed-effect model was used in the absence of between-study heterogeneity $\left(P>0.10\right.$ or $I^{2}<$ 
$50 \%)$. As sensitivity analysis to confirm the robustness of our results, we performed a subgroup analysis for the control arm of no private health insurance in order to distinguish individuals with no insurance from individuals with public insurance.

\section{Results}

\section{Study selection}

A total of 8727 articles were selected by searching the selected electronic databases, and an additional five records were identified by cross-checking the reference lists of retrieved studies or relevant reviews. After excluding duplicates and screening titles and abstracts, we obtained 181 articles for full-text review. We eliminated 155 papers from among the 181 originally identified, based on our inclusion and exclusion criteria. Ultimately, 26 articles were included in the analysis (Fig. 1).

\section{Study characteristics}

The basic characteristics and target outcomes of included studies are listed in Table 1. All included articles $(n=26)$ were observational studies, in the form of abstract $(n=6)$ or full report $(n=20)$. The studies included in the meta-analysis had been conducted in the United States $(57.7 \%, 15 / 26)$, Brazil $(11.5 \%, 3 / 26)$, South Korea (7.7\%, 2/26), Australia $(7.7 \%, 2 / 26)$, India $(7.7 \%, 2 / 26)$, Japan $(3.8 \%, 1 / 26)$, and Germany $(3.8 \%, 1 / 26)$. The study populations ranged from healthy controls to patients with specific diseases or medical conditions. Among the 26 studies included, 13 (50\%) focusing on the comparison between private insurance and both no insurance and public insurance, 8 (30.8\%) on the comparison between private insurance and no public insurance, and 5 studies (19.2\%) compared private insurance with a lack of insurance.

\section{Risk of bias}

We evaluated risk of bias for all full reports included in the meta-analysis $(n=20)$ with the ROBINS I tool. We did not assess the risk of bias in abstracts because there was insufficient information for the evaluation of methodological quality. Figure 2a shows the risk of bias for each cohort. Evaluations for each domain are shown in Fig. 2b. These figures did not include studies reported as abstracts only.

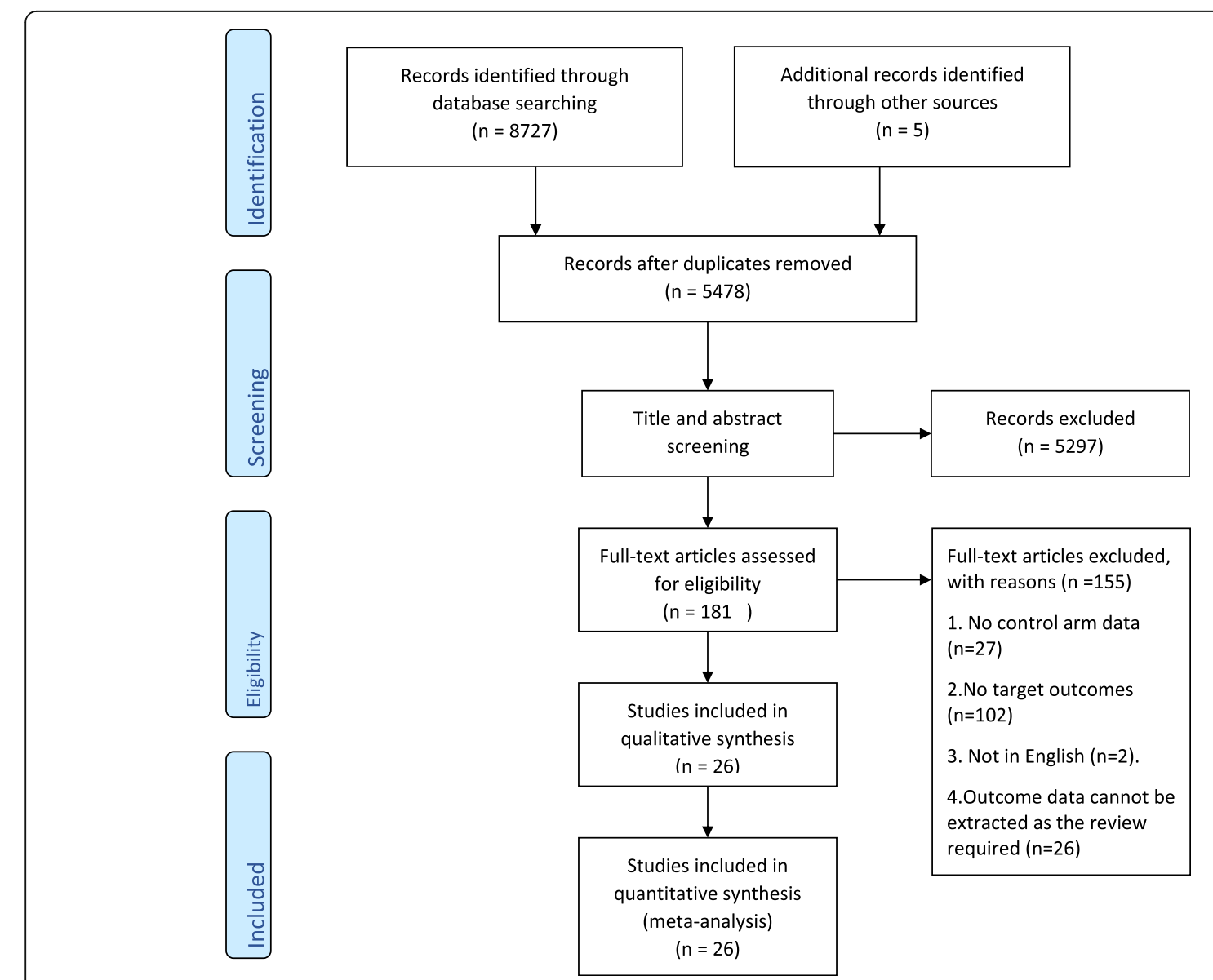

Fig. 1 PRISMA flow diagram detailing the search strategy and results 


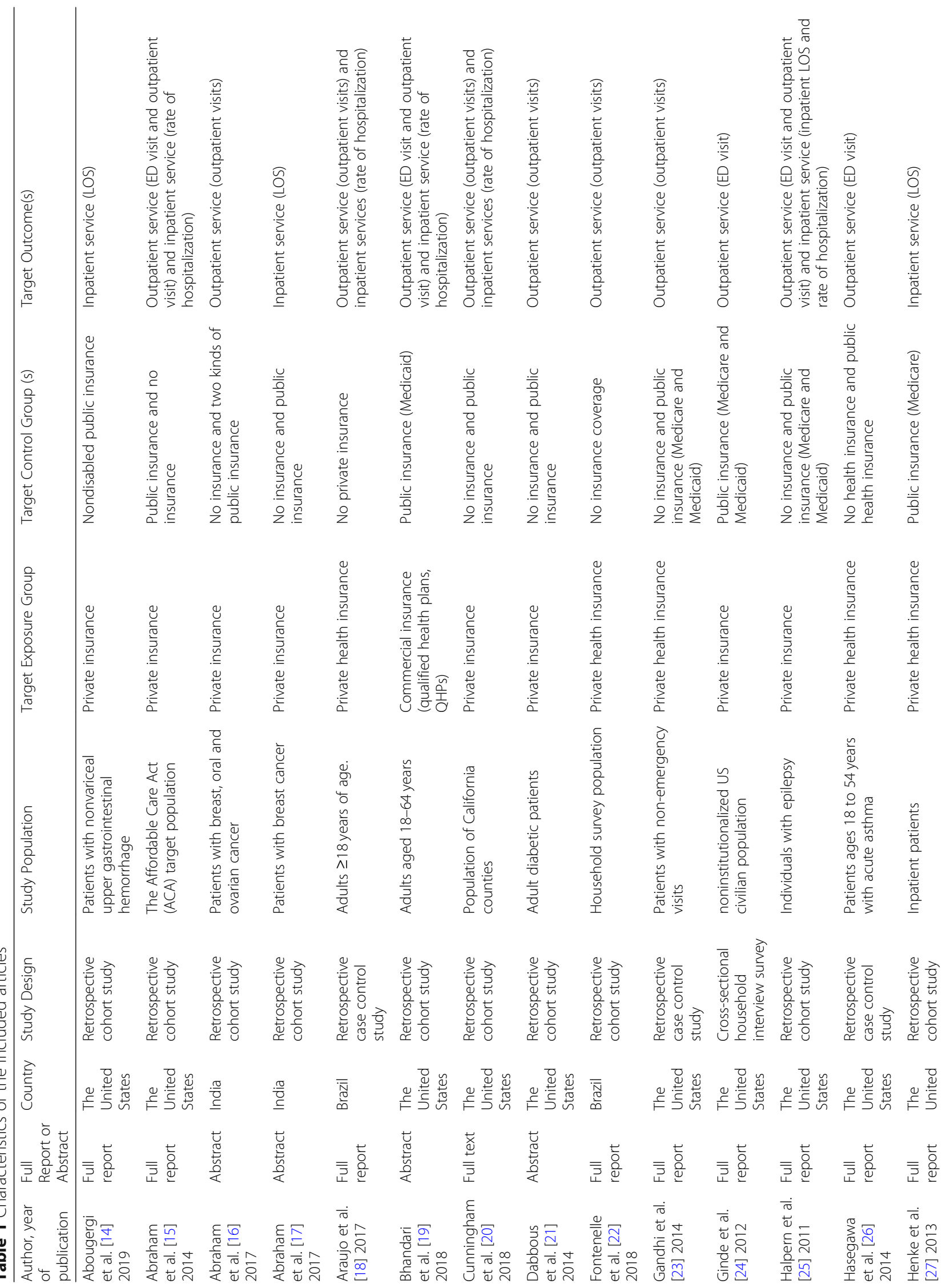




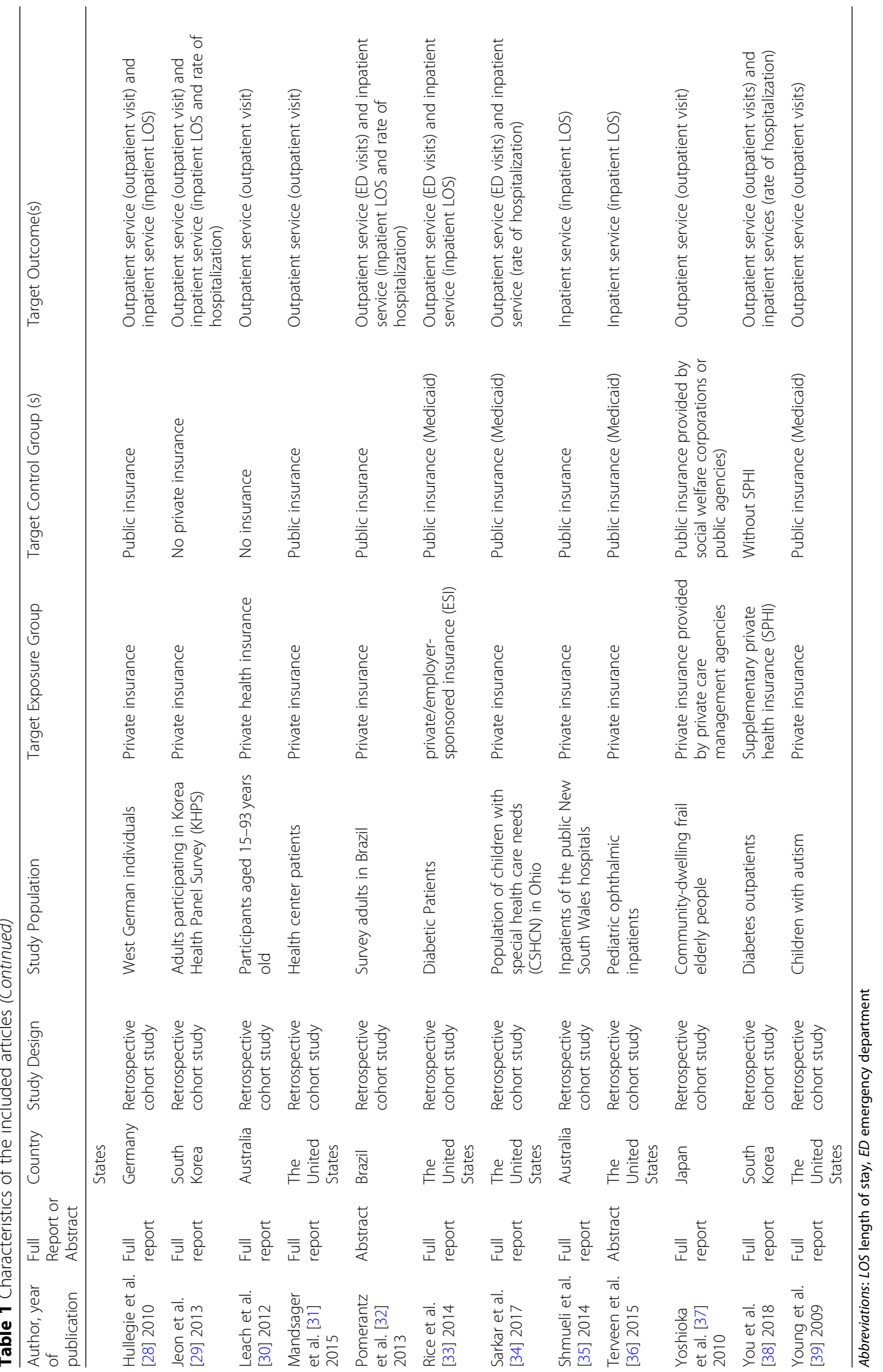




\section{Utilization of outpatient services}

All detailed data extraction results can be found in Additional file 1.

\section{ED visits}

We used the data from 5 studies, which collectively included $>500,000,000$ participants, to determine the odds ratio $(\mathrm{OR})$ for a comparison of the rates of $E D$ visits among people with private insurance, compared to people without private insurance $[15,19,20,25$, 34]. The pooled results yielded an OR of 1.01 (95\% CI 0.58-1.76) (Fig. 3a). There was no significant difference between people with private insurance and people without private insurance in the rate of ED visits. The results of subgroup analysis showed that this OR was similar for people with public insurance and people with no insurance (Fig. 4a).

Three included studies, which included 285,570 participants, reported the percentage of study participants who had visited the ED [24, 26, 32]. The proportion of those with private insurance who visited the ED was similar to the proportion of people without private insurance who visited the ED. The OR for pooled results was 0.65 (95\% CI $0.27-1.60)$. See Fig. 3b.

The results of subgroup analysis (Fig. 4b) showed that there was no significant difference between the percentage of people with private insurance who visited the ED and either those with public insurance or those with no insurance at all.

\section{Rate of outpatient office visits}

We used the data from 7 studies, which included 120,887 participants, to determine the mean difference in the rate of outpatient office visits between people with private insurance and people without private insurance. After pooling the results, the mean difference was -0.19 (95\% CI -0.29 to -0.09$)$ (see forest plot in Fig. 3c). People with private insurance were significantly less likely to visit the hospital as outpatients than people without private insurance. According to the subgroup analysis, people with private insurance were less likely to visit the outpatient office, compared to people with public insurance, and also compared to people without insurance $(P<$ 0.05) (see forest plot in Fig. 4c). In Additional file 1, we present the data pertaining to outpatient visits that could not be included in the meta-analysis (9 articles) $[15-18,21,25,32,37,38]$. The favorable results (more outpatient visits) for both people with private insurance and people without private insurance were reported.

\section{Utilization of inpatient services Inpatient LOS}

We used the data from 4 studies, which included 304, 431 participants, to determine the mean difference in LOS (days) between people with private insurance and people without private insurance [27-29,33]. The mean difference in pooled results was 2.01 (95\% CI -0.15 to 4.17, Fig. 3d). There was no significant difference between people with private insurance and people without private insurance in terms of inpatient LOS.

According to the subgroup analysis (Fig. 4d), compared to people with public insurance, people with private insurance were more likely to stay longer in the hospital (mean difference (days) $=2.82,95 \%$ CI $0.38-5.27)$. While there was only one study left for compared to people without private insurance with the results of mean difference of LOS $(-1.30,95 \%$ CI -2.15 to -0.45 ), which means the favorite result (longer of LOS) for people without private insurance.

In Additional file 1, we list the data for the mean difference in LOS from 6 articles that could not be included in the meta-analysis $[14,16,19,25,35,36]$. Favorable results (longer LOS) for both people with private insurance and people without private insurance were reported.

\section{Rate of hospitalization}

We used the data from 7 studies, which included > $500,000,000$ participants in determining the OR for the rate of hospitalization among those with private insurance, compared with those without private insurance $[15,20,25,29,32,34,38]$. The OR for the pooled results was 1.00 (95\% CI 0.58-1.70) (see forest plot in Fig. 3e). There was no significant difference in the rate of hospitalization between people with private insurance and people without private insurance.

According to the subgroup analysis (Fig. 4e), those with public insurance and those with private insurance had similar rates of hospitalization $(\mathrm{OR}=0.72$, 95\% CI 0.33-1.60). Compared to people with no insurance, people with private insurance were more likely to be hospitalized (OR 1.67; 95\% CI, 1.182.36).

\section{The certainty of the evidence (GRADE)}

The certainty of the evidence ranged from low to moderate. The observational study design meant the GRADE rating started as moderate certainty (Table 2), and almost all studies (except Abougergi et al. 2019) were missing data. Furthermore, we considered it likely that possible biases and confounding factors would have had a significant impact on the results presented in abstract form only. 


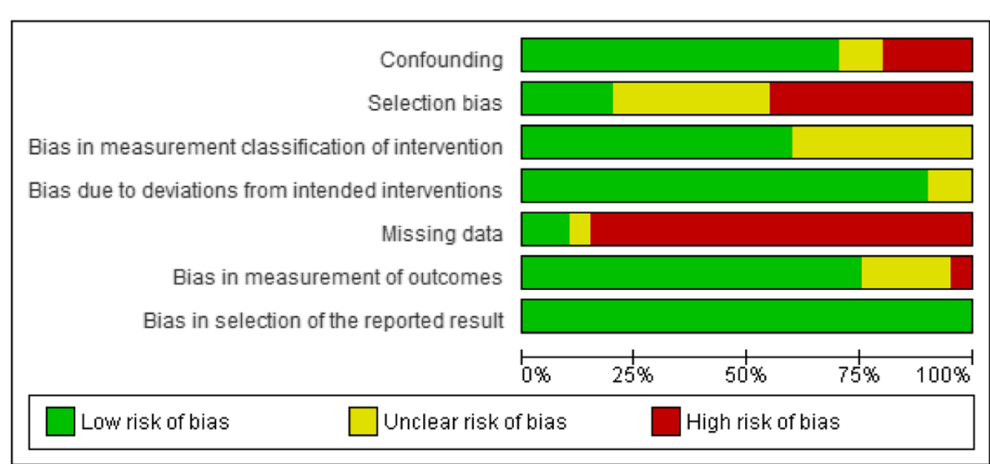

A

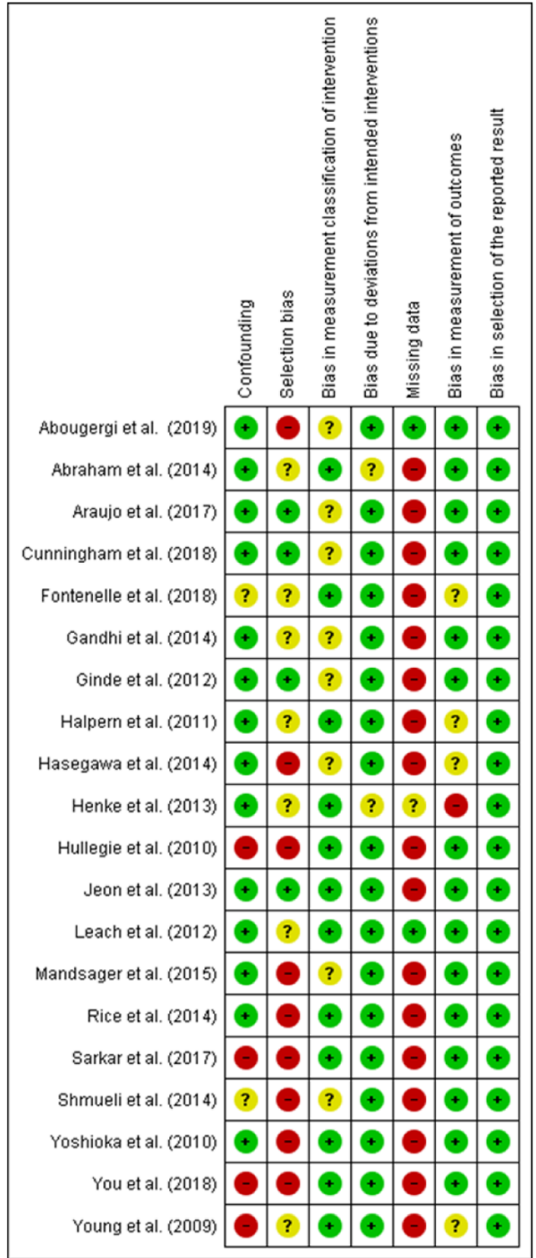

B

Fig. 2 Risk of bias assessment. a Risk of bias graph: review authors' judgements about each risk of bias item presented as percentages across all included full reported studies $(n=20)$. b Risk of bias summary: review authors' judgements about each risk of bias item for each included full reported study 


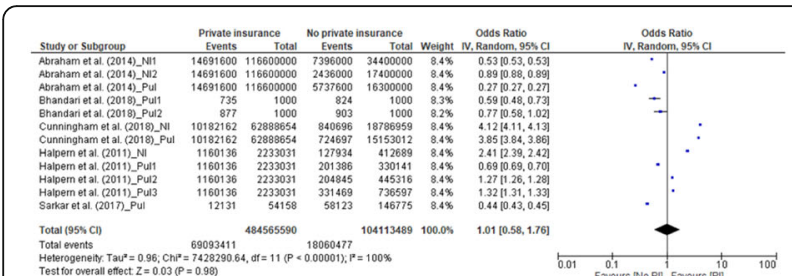

A

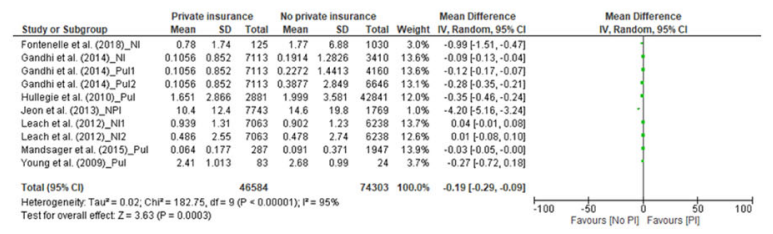

C

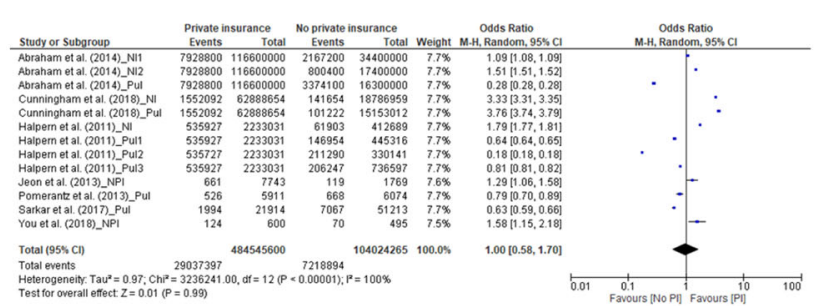

$E$

Fig. 3 Forest plots of the total pooling results. a for comparison of the rates of emergency department visits between private insurance and no private insurance. $\mathbf{b}$ for comparison of the percentages of emergency department visits between those with private insurance and those without private insurance. $\mathbf{c}$ for comparison of the rates of outpatient office visits between private insurance and no private insurance. $\mathbf{d}$ for comparison of the rates of outpatient office visits between private insurance and no private insurance. e for comparison of the length of inpatient stay (days) between those with private insurance and those without private insurance

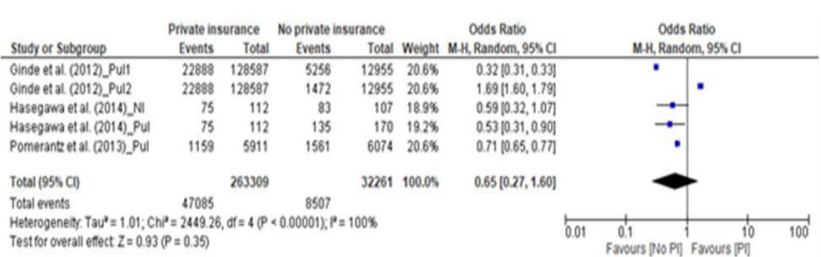

B

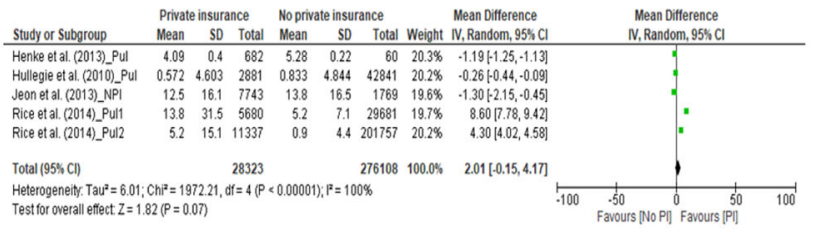

D

\section{Discussion}

In this systematic review, we investigated whether people with private insurance were more likely to utilize health care than those without private insurance. According to the results of the meta-analysis, the utilization was similar between those with and those without private health insurance. For the target outcome of outpatient office visits, people with private insurance were less likely to visit the outpatient office than people without private insurance (mean difference $=-0.19(95 \% \mathrm{CI}-0.29$ to $0.09)$ ). In theory, people with private insurance should have more access to health care. However, our results indicate that there was no significant increase in the consumption of healthcare services among individuals with private health insurance. In one of the dimensions examined, those with private health insurance coverage actually used fewer of the health care available to them. One possible explanation is that the utilization of medical services was more directly correlated with the need for the service than with insurance coverage, as suggested by previous studies [4042]. Private health insurance coverage does not appear to increase the utilization of health care and may ease the financial burdens on patients and social health insurance plans.

The results of subgroup analysis to identify differences between those without insurance and those with public insurance showed that most results were consistent with the total pooled results. For LOS, people with private insurance were more likely to stay longer in the hospital, compared to people with public insurance (mean difference (days) $=2.82,95 \%$ CI $0.38-5.27)$. With regard to the rate of hospitalization, compared to people without any insurance, people with private insurance were more likely to be hospitalized (OR 1.67; 95\% CI, 1.18-2.36). As inpatient services are more tightly linked to medical necessity than outpatient services, these results reflect the potential for private insurance to relieve patients' financial burden.

To our best knowledge, this systematic review is the first review to assess the impact of private insurance coverage on the utilization of health care across the globe. This study strictly followed the standards for 


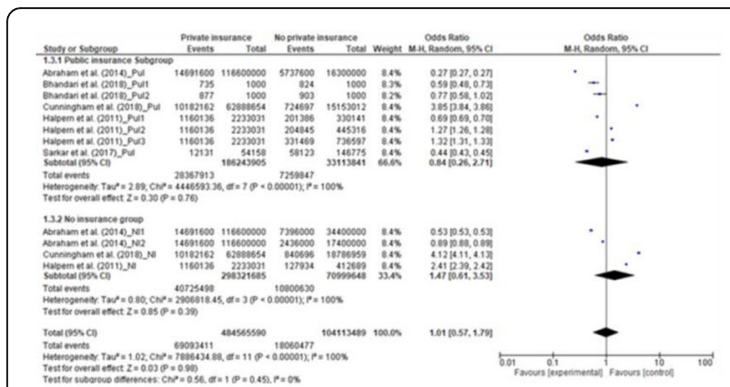

A

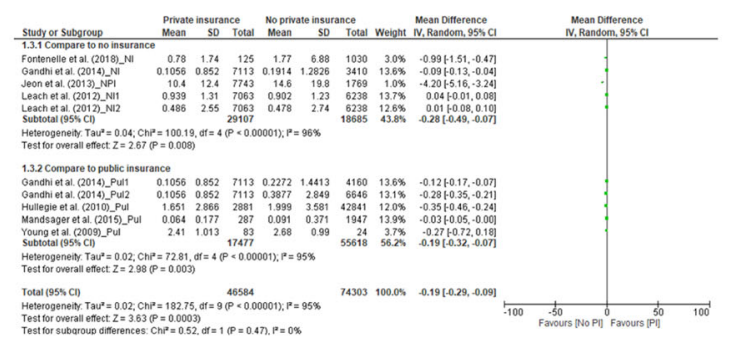

$\mathrm{C}$

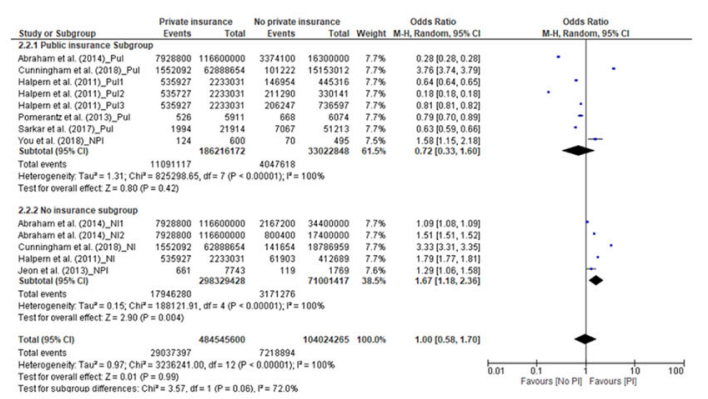

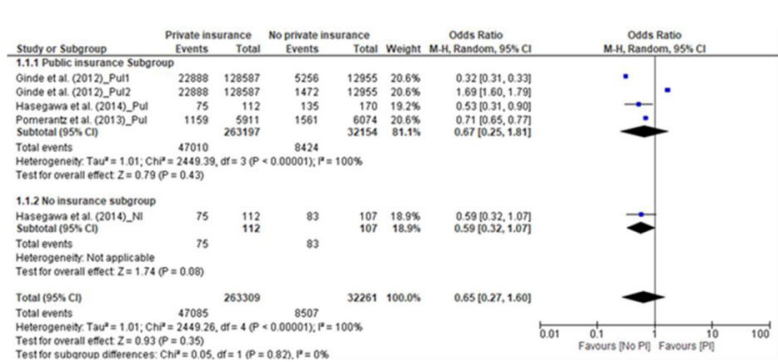

B

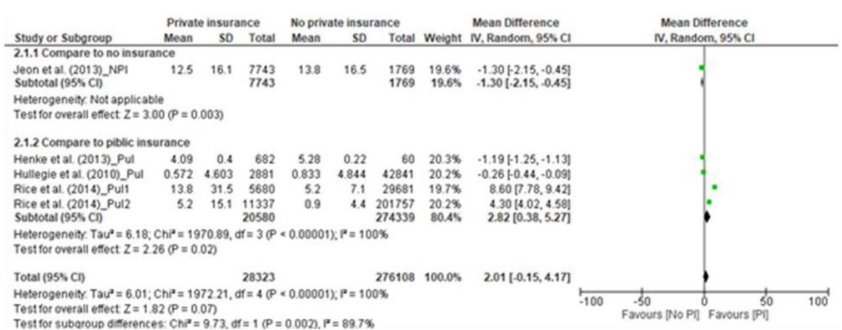

$\mathrm{D}$

E

Fig. 4 Forest plots of subgroup analysis according to the control of public insurance and no health insurance. a for comparison of the rates of emergency department visits between private insurance and no private insurance. $\mathbf{b}$ for comparison of the percentages of emergency department visits between those with private insurance and those without private insurance. $\mathbf{c}$ for comparison of the rates of outpatient office visits between private insurance and no private insurance. $\mathbf{d}$ Comparison of the length of inpatient stay (days) between those with private insurance and those without private insurance. e Comparison of the rates of hospitalization between individuals with private insurance and those with no private insurance

systematic reviews, including explicit eligibility criteria, duplicated independent assessments of eligibility, and a comprehensive literature search. One limitation of this study was that more than half of the included studies were conducted in the United States, which restricted the external validity of the results. Another limitation of this review is that the results may have been confounded by selection bias due to divergences in methodology among health care systems. Next, the evidence of this present study has temporal limitations. Studies on this topic were conducted prior to 2010. However, we restricted the search period to years from 2010 onward in order to focus our investigation on current insurance policy. Finally, as there is no standardized tool for the assessment of abstract quality, all abstracts included in the review were not graded in terms of quality. This fact may limit the ability of other researchers to extrapolate from the results reported here. Additional studies will be necessary to explore these issues.

\section{Conclusion}

People with private insurance did not increase their utilization of health care (outpatient services), compared to those without private insurance. Private health insurance coverage may ease the financial burdens on patients and on the public health insurance system. 


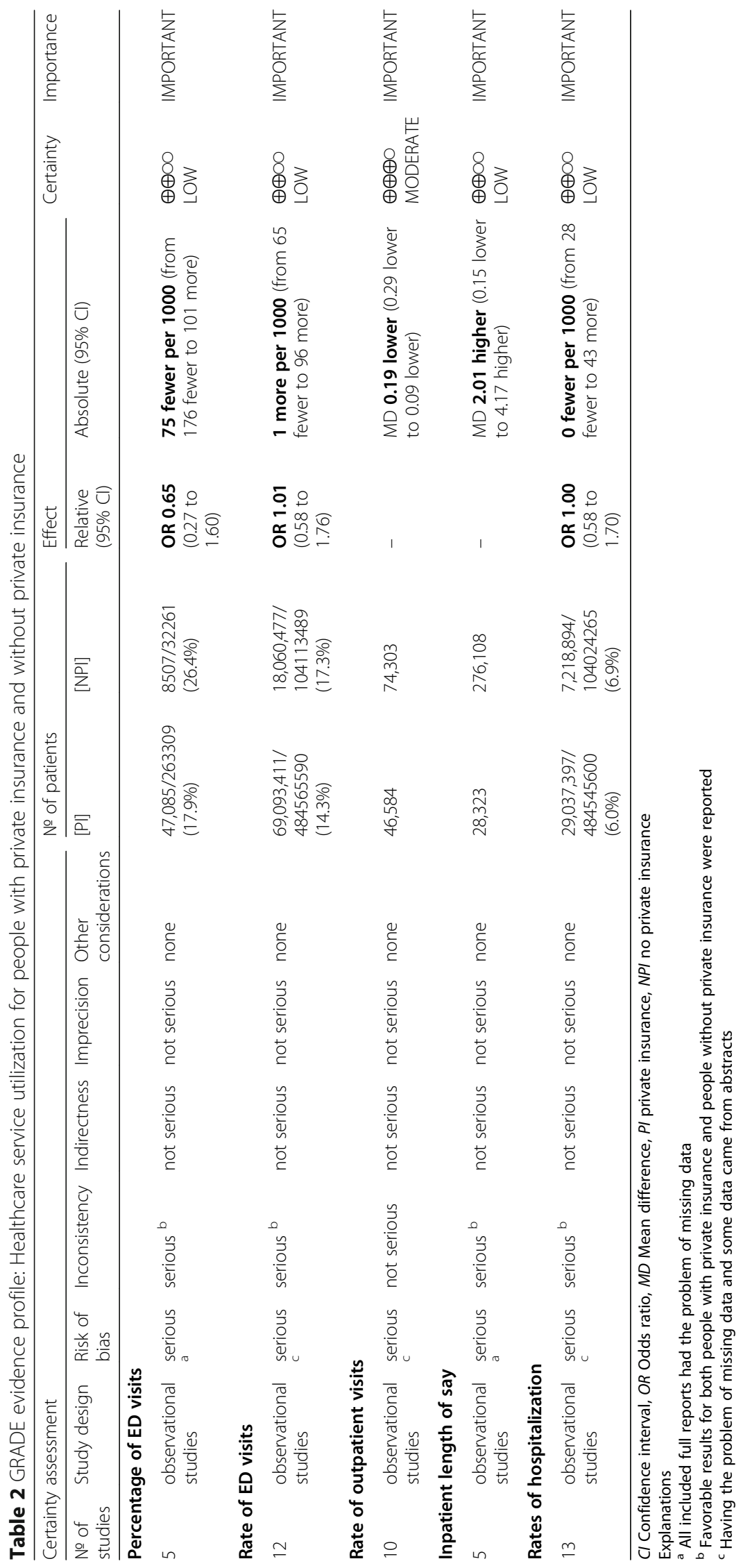




\section{Supplementary information}

Supplementary information accompanies this paper at https://doi.org/10. 1186/s12889-020-08861-9.

\section{Additional file 1}

\section{Abbreviations}

PRISMA: Preferred Reporting Items for Systematic Reviews and MetaAnalyses; CENTRAL: Cochrane Central Register of Controlled Trials; ED: Emergency department; LOS: Length of stay; Cls: Confidence intervals; OR: Odds ratio

\section{Acknowledgments}

I am grateful to my department leaders and my family for their great encouragement, support and help to this project.

\section{Authors' contributions}

CZ was responsible for the search strategy, designing, testing of the data extraction form, and writing the initial draft. $\mathrm{YL}$ and $\mathrm{XW}$ provided input for the design, analyses and reporting. XW was responsible for the conception, designing of the survey and critical review the final draft. CZ and CF were involved in designing and testing of the data extraction form. CZ, CF, YS and RF contributed to quality checking of the data and the manuscript writing. All authors contributed to the protocol and approved the final manuscript.

\section{Funding}

This research did not receive any specific grant from funding agencies in the public, commercial, or not-for-profit sectors.

\section{Availability of data and materials}

The datasets used during the current study are available from the corresponding author on reasonable request.

\section{Ethics approval and consent to participate}

The present project is a systematic review based on review of publicly reported literature, which does not require ethics review.

\section{Consent for publication}

Not applicable.

\section{Competing interests}

The authors declare that they have no competing interests.

Received: 20 January 2020 Accepted: 6 May 2020

Published online: 23 July 2020

\section{References}

1. Tamblyn R, Laprise R, Hanley JA, Abrahamowicz M, Scott S, Mayo N, et al. Adverse events associated with prescription drug cost-sharing among poor and elderly persons. Jama. 2001;285:421-9.

2. Manning WG, Newhouse JP, Duan N, Keeler EB, Leibowitz A, Marquis MS. Health insurance and the demand for medical care: evidence from a randomized experiment. Am Econ Rev. 1987;77:251-77.

3. Fontenelle LF, Sarti TD, Camargo MBJ, Maciel ELN, Barros AJD. Utilization of the Brazilian public health system by privately insured individuals: a literature review. Cad Saude Publica. 2019;35:e00004118.

4. Barnett JC, Vornovitsky MS. Health insurance coverage in the United States: 2015. Washington, DC: US Government Printing Office; 2016.

5. Iglehart JK. Canada's health care system. In: Mass Medical Soc; 1986

6. Sohn M, Jung M. Effects of public and private health insurance on medical service utilization in the National Health Insurance System: national panel study in the Republic of Korea. BMC Health Serv Res. 2016;16:503.

7. Bodenheimer T. Should we abolish the private health insurance industry? Int J Health Serv. 1990;20:199-220.

8. Moher D, Liberati A, Tetzlaff J, Altman DG. Preferred reporting items for systematic reviews and meta-analyses: the PRISMA statement. Int J Surg. 2010:8:336-41.

9. Carrasquillo O. Health Care Utilization. In: Gellman MD, Turner JR, editors Encyclopedia of Behavioral Medicine. New York: Springer New York; 2013. p. 909-10
10. Sterne JA, Hernan MA, Reeves BC, Savovic J, Berkman ND, Viswanathan M, et al. ROBINS-l: a tool for assessing risk of bias in non-randomised studies of interventions. Bmj. 2016;355:14919.

11. Guyatt GH, Oxman AD, Vist GE, Kunz R, Falck-Ytter Y, Alonso-Coello P, et al. GRADE: an emerging consensus on rating quality of evidence and strength of recommendations. Bmj. 2008;336:924-6.

12. Manager R: Version 5.0. In.: The Nordic Cochrane Centre, The Cochrane Collaboration Copenhagen, Denmark; 2008.

13. DerSimonian R, Laird N. Meta-analysis in clinical trials. Control Clin Trials. 1986;7:177-88.

14. Abougergi MS, Avila P, Saltzman JR. Impact of insurance status and race on outcomes in Nonvariceal upper gastrointestinal hemorrhage: a nationwide analysis. J Clin Gastroenterol. 2019;53:E12-E8.

15. Abraham JM. How might the affordable care Act's coverage expansion provisions influence demand for medical care? Milbank Q. 2014:92:63-87.

16. Abraham PS, Greene M. The impact of different types of payer on healthcare resource utilization and costs among cancer patients in India. Value Health. 2017;20(5):A131.

17. Abraham PS, Greene M, Eguale T, Rodriguez-Monguio R, Seoane-Vazquez E. Impact of payer on healthcare resource utilization and costs among breast cancer patients in India. Value Health. 2017:20(5):A128.

18. Araujo MEA, Silva MT, Galvao TF, Pereira MG. Prevalence of health services usage and associated factors in the Amazon region of Brazil: A populationbased cross-sectional study. BMJ Open. 2017;7(11) (no pagination).

19. Bhandari NR, Chopra DA, Kathe N, Moore G, Lewis K, Li C, et al. Contrasting utilization of inpatient hospitalizations and emergency department visits between medicaid and commercially insured arkansans. Value Health. 2018; 21(Supplement 1):S131.

20. Cunningham $P$, Sheng $Y$. Trends in preventable inpatient and emergency department utilization in California between 2012 and 2015. Med Care. 2018;56:544-50

21. Dabbous FM, Dorey J, Thokagevistk K, Toumi M. Impact of health insurance status on health care resource utilization among diabetic patients in the United States. Value Health. 2014;17(3):A252.

22. Fontenelle LF, Camargo MBJ, Bertoldi AD, Goncalves H, Maciel ELN, Barros AJD. Utilization of basic health units of FHS according to private health insurance. Revista de Saude Publica. 2018;52:55.

23. Gandhi SO, Grant LP, Sabik LM. Trends in nonemergent use of emergency departments by health insurance status. Med Care Res Rev. 2014;71:496521.

24. Ginde AA, Lowe RA, Wiler JL. Health insurance status change and emergency department use among US adults. Arch Intern Med. 2012;172: 642-7.

25. Halpern MT, Renaud JM, Vickrey BG. Impact of insurance status on access to care and out-of-pocket costs for U.S. individuals with epilepsy. Epilepsy Behav. 2011;22:483-9.

26. Hasegawa K, Sullivan AF, Tovar Hirashima E, Gaeta TJ, Fee C, Turner SJ, et al. A Multicenter Observational Study of US Adults with Acute Asthma: Who Are the Frequent Users of the Emergency Department? J Allergy Clin Immunol: In Pract. 2014;2:733-40.e3.

27. Henke RM, Maeda JL, Marder WD, Friedman BS, Wong HS. Medicare and commercial inpatient resource use: impact of hospital competition. Am J Manag Care. 2013:19:e238-e48.

28. Hullegie P, Klein TJ. The effect of private health insurance on medical care utilization and self-assessed health in Germany. Health Econ. 2010;19:1048-62.

29. Jeon B, Kwon S. Effect of private health insurance on health care utilization in a universal public insurance system: a case of South Korea. Health Policy. 2013;113:69-76

30. Leach LS, Butterworth $P$, Whiteford $H$. Private health insurance, mental health and service use in Australia. Aust N Z J Psychiatry. 2012:46:468-75.

31. Mandsager $P$, Lebrun-Harris LA, Sripipatana A. Health center patients' insurance status and healthcare use prior to implementation of the affordable care act. Am J Prev Med. 2015;49:545-52.

32. Pomerantz D, Machado M, DiBonaventura $M$. The impact of private versus public insurance on health status, work productivity and health care utilization for individuals residing in Brazil. Value Health. 2013;16(3):A76.

33. Rice JB, Desai U, Cummings AK, Birnbaum HG, Skornicki M, Parsons NB. Burden of diabetic foot ulcers for medicare and private insurers. Diabetes Care. 2014;37:651-8.

34. Sarkar M, Earley ER, Asti L, Chisolm DJ. Differences in health care needs, health care utilization, and health care outcomes among children with 
special health care needs in Ohio: a comparative analysis between Medicaid and private insurance. J Public Health Manag Pract. 2017;23:e1-9.

35. Shmueli A, Savage E. Private and public patients in public hospitals in Australia. Health Policy. 2014;115:189-95.

36. Terveen DC, Hsu BS, Tufty GT. The impact of insurance status on hospital resource utilization in pediatric ophthalmic inpatients. J AAPOS. 2015;19(4): e65.

37. Yoshioka Y, Tamiya N, Kashiwagi M, Sato M, Okubo I. Comparison of public and private care management agencies under public long-term care insurance in Japan: a cross-sectional study. Geriatr Gerontol Int. 2010;10:4855.

38. You CH, Choi JH, Kang S, Oh EH, Kwon YD. Association between supplementary private health insurance and visits to physician offices versus hospital outpatient departments among adults with diabetes in the universal public insurance system. PLoS ONE. 2018:13(4) (no pagination).

39. Young A, Ruble L, McGrew J. Public vs. private insurance: cost, use, accessibility, and outcomes of services for children with autism spectrum disorders. Res Autism Spectr Disord. 2009;3:1023-33.

40. Geil P, Million A, Rotte R, Zimmermann KF. Economic incentives and hospitalization in Germany. J Appl Econ. 1997;12:295-311.

41. Riphahn RT, Wambach A, Million A. Incentive effects in the demand for health care: a bivariate panel count data estimation. J Appl Econ. 2003;18: 387-405.

42. Jürges $\mathrm{H}$. Health insurance status and physician behavior in Germany. Schmollers Jahr. 2009;129:297.

\section{Publisher's Note}

Springer Nature remains neutral with regard to jurisdictional claims in published maps and institutional affiliations.

Ready to submit your research? Choose BMC and benefit from:

- fast, convenient online submission

- thorough peer review by experienced researchers in your field

- rapid publication on acceptance

- support for research data, including large and complex data types

- gold Open Access which fosters wider collaboration and increased citations

- maximum visibility for your research: over $100 \mathrm{M}$ website views per year

At BMC, research is always in progress.

Learn more biomedcentral.com/submissions 